\title{
Touchpad Monitored Car
}

\author{
Priyanka Dalvi ${ }^{1}$, Girish Joshi ${ }^{2}$ \\ ${ }^{1}$ (Asst. Professor, Department of Electronics \& Telecommunication, MPCOE, Velneshwar, Mumbai University, \\ India) \\ ${ }^{2}$ (Asst. Professor, Department of Mechanical Engineering, MPCOE, Velneshwar, Mumbai University, India)
}

\begin{abstract}
In the present study, innovative idea of touchpad controlled vehicle and its real life implication is described. Generally in such system touchpad is interfaced with ADC [0808/MCP 3208] which gives the coordinates of the points touch by the user on touchpad. But in this research work Programmable Intercombination Circuit (PIC) [18F4550/18C4550] is used purposefully instead of ADC as input signal were in analog which required converting into digital signal. PIC can be used to interface touchpad and also perform serial port programming far better than ADC. PIC gives output to 8051 microcontroller which uses keil software program in $C$ for input and output programming. Test drive was done to crosscheck the performance of touchpad as well as vehicle and it was observed that car was running at corresponding given location directed by touchpad at al instants.
\end{abstract}

Keywords - Touch screen, PIC 18F4550, Motor driverL293D, RF Transmitter \& Receiver, Keil software.

\section{INTRODUCTION}

In this work, it is aimed to replace not only complicated driving arrangement but also make enable to drive vehicle from inside as well as from outside. The concept of touchpad is acts as a remote of vehicle, which helped it to drive the car anywhere with and without driver inside it.

Touchpad controlled vehicle has various applications; like this can be use in future as a robot car, as a devise which can be used to identify location of hidden terrorist. Also if a sensor can be placed on it, then it works as movable sensing device to study wild nature thoroughly. Further this can be adopt by putting high quality camera on it and can be use where manually going is not possible [1].

\subsection{Touchpad Remote Control}

\section{SYSTEM DESIGN}

This part consists 4 major blocks as shown in the block diagram, given in Fig. 01. The touchpad is interfaced to an ADC (0808 or MCP 3208) which gives the co-ordinates of the point touched by the user on the touchpad. These co-ordinates are fetched by necessary action and transmit a unique character (say 1 for forward, 2 for backward, 3 for left, 4 for left and 5 for stop) serially through serial port. An RF transmitter ( $315 \mathrm{MHz}$ or $433 \mathrm{MHz}$ ) is connected to the serial port which transmits these characters through an antenna.

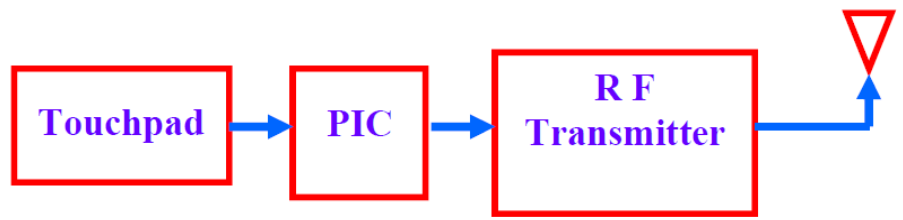

Fig. 1 Block diagram of transmitter

\subsection{Transmitter section}

In this work, the main signal was in analog form which needs to be converted into digital. For this programmable intercombination circuit (PIC) was used instead of analog to digital convertor (ADC). It is advantageous to use programmable intercombination circuit (PIC) over analog to digital convertor (ADC) because PIC can be used to interface touchpad and also perform serial port programming. PIC gives it output to 8051 which uses keil software programmed in C for input, output programming. The output of 8051 is performed on looping basis. The output is transmitted through RF transmitter.

\subsection{Vehicle}

This part also consists of 4 major blocks as shown in Fig. 02. The RF receiver receives the serial data (character 1, 2, 3, 4, or 5) transmitted from the remote control circuit and gives it to microcontroller 8051 . Depending upon the data received serially 8051 takes move the vehicle, 8051 controls the two $12 \mathrm{~V}$ DC motors connected to it. The 8051 cannot directly drive the DC motors because it lacks sufficient current. Therefore, a 
motor driver IC (L293D or ULN2803) is used to drive the motors. The circuit diagram for Vehicle is shown in Fig. 03.

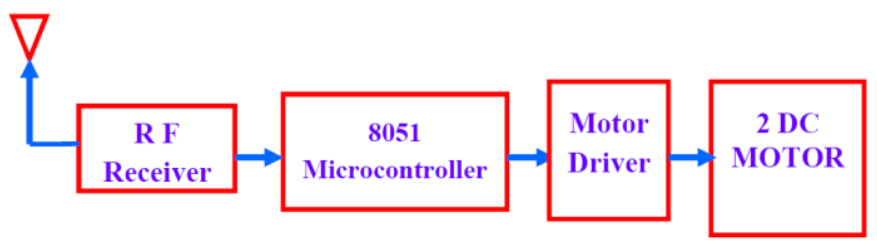

Fig. 2 Block diagram of receiver

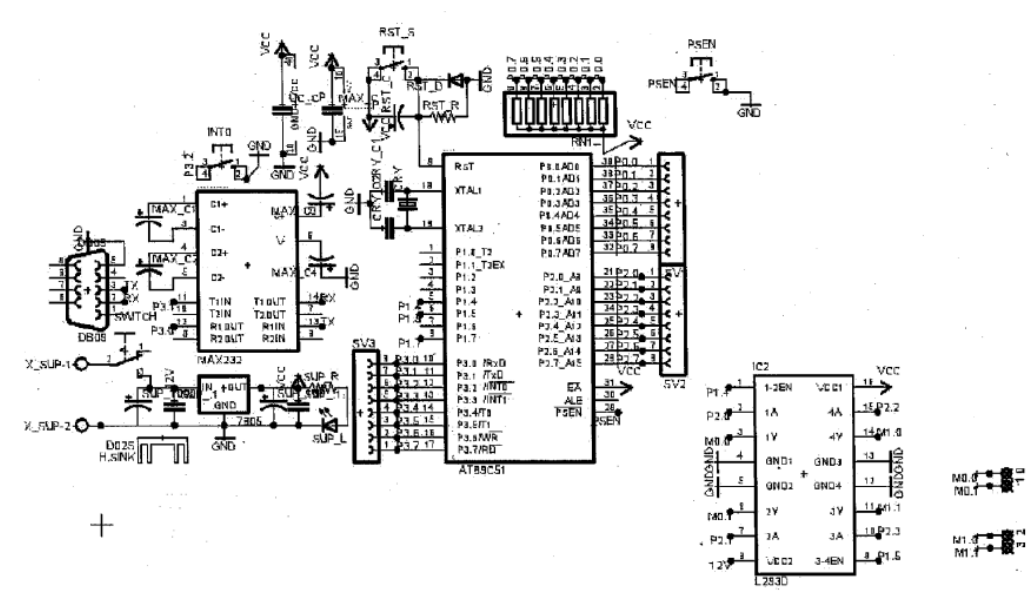

Fig. 3 Circuit diagram for vehicle

\subsection{Receiver section}

The transmitted output is received through RF receiver which is connected to another 8051. Here 8051 is used to interface driver and motors. The gist of the project is hidden in this part. The vehicle is operated on the motors which are connected through driver to the 8051. The motion of the vehicle is based on the rotation of the DC motors. The motion of the vehicle is as given in Table 01.

Table 0 motion of vehicle according to motor sequence

\begin{tabular}{lll}
\hline Motion & DC motor-1 & DC motor-2 \\
\hline FORWARD & FORWARD & FORWARD \\
BACKWAED & BACKWAED & BACKWAED \\
RIGHT & FORWARD & BACKWAED \\
LEFT & BACKWAED & FORWARD \\
STOP & NO MOTION & NO MOTION \\
\hline
\end{tabular}

There are four connections of two motors to 8051 microcontroller. Out of these four connections, two are connected to ground GND and two are connected to VCC supply. For this interfacing any port of 8051 can be used. According to mentioned combination vehicle moves as per given motion command. Based on this connection various combinations are possible according to which the vehicle is set into motion in particular direction. Interfacing of PIC port with motion of vehicle is given as in Table 02

Table 02 Interfacing of PIC port with motion of vehicle

\begin{tabular}{lcccc}
\hline MOTION & PORT 1 & PORT 2 & PORT 3 & PORT 4 \\
\hline FORWARD & 1 & 0 & 1 & 0 \\
BACKWARD & 0 & 1 & 0 & 1 \\
LEFT & 1 & 0 & 0 & 1 \\
RIGHT & 0 & 1 & 1 & 0 \\
STOP & 0 & 0 & 0 & 0 \\
\hline
\end{tabular}




\subsection{Touch Screen}

\section{HARDWARE USED}

A touch screen is a two dimensional sensing device which consist of two sheets of material separated slightly by spacer as shown in Fig. 04. Resistive coating is applied on top film as well as on bottom glass. Polyethylene (PET) acts as a flexible top layer whereas resistive coating on bottom of glass acts as flexible bottom layer. These two sheets are coated with a metal compound called INDIUM TIN OXIDE (ITO) usually. The ITO is thinly and uniformly sputtered onto both; the glass as well as on the PET layer. Tiny bumps called spacer dots are then added to the glass side, on top of the ITO coating, to keep the PET film from sagging, causing an accidental or false touch [2].

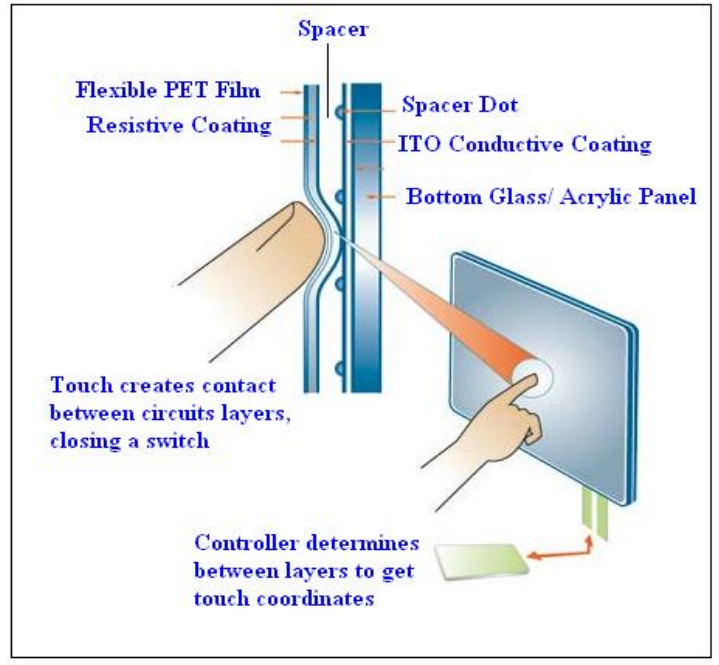

Fig. 4 Typical analog touch screen construction

When the PET film is press down the two resistive surfaces meet each other. The portion of this meeting can be read by touch screen controller circuit. ' $\mathrm{X}$ ' touch position can be achieved by connecting the controller sets PIN 4 to $+5 \mathrm{~V}$ and PIN 2 to the ground (o V). At this condition PIN 1 left unconnected. The controller uses PIN 3 to read the voltage where the top layer meets the bottom layer. The controller converts the voltage to a number (data) and sends it to the host computer for further operation. Similarly to get the ' $\mathrm{Y}$ ' touch position the controller sets PIN 1 to $+5 \mathrm{~V}$ and PIN 3 grounded. In this case PIN 2 is left unconnected. For capturing ' $\mathrm{Y}$ ' touch position the controller uses PIN 3 to read the voltage where the top layer meets the bottom layer. Again, the controller converts the voltage to a number (data) and sends it to the host computer [3]. The capturing of ' $\mathrm{X}$ ' and ' $\mathrm{Y}$ ' is as shown n Fig. 05.

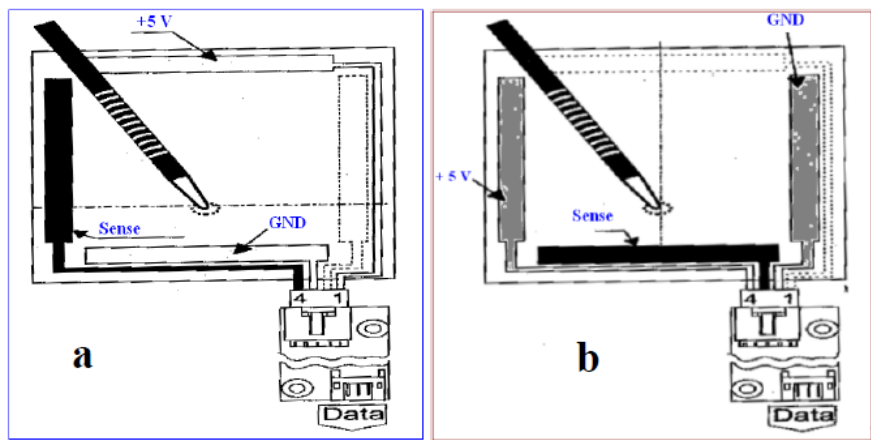

Fig. 5 Capturing (a) "X" Touch, (b) "Y" Touch

\subsection{PIC (18F4550/18C4550)}

In fact a PIC microcontroller is an amazingly fully featured processor with internal RAM, EEROM FLASH memory and peripherals. One of the smallest ones occupies the space of a 555 timer but has a ten bit ADC, $1 \mathrm{~K}$ of memory, two timers; high current input output ports a comparator a watch dog timer. One of the most useful features of a PIC microcontroller is, it is re- programmable as it uses flash memory. ICSP serial interface built also use into each PIC microcontroller for programming. PIC microcontroller can be programmed by using assembler or a high level language and it is recommend using high level language such as $\mathrm{C}$ as it is 
much easier to use. Flash memory is the program storage area and gives the most important benefit for using PIC microcontroller. Device used in this research was re-programmed up to 100,000 times as it uses flash memory $[4,5]$. Circuit diagram of PIC used in this work is given in Fig. 06.

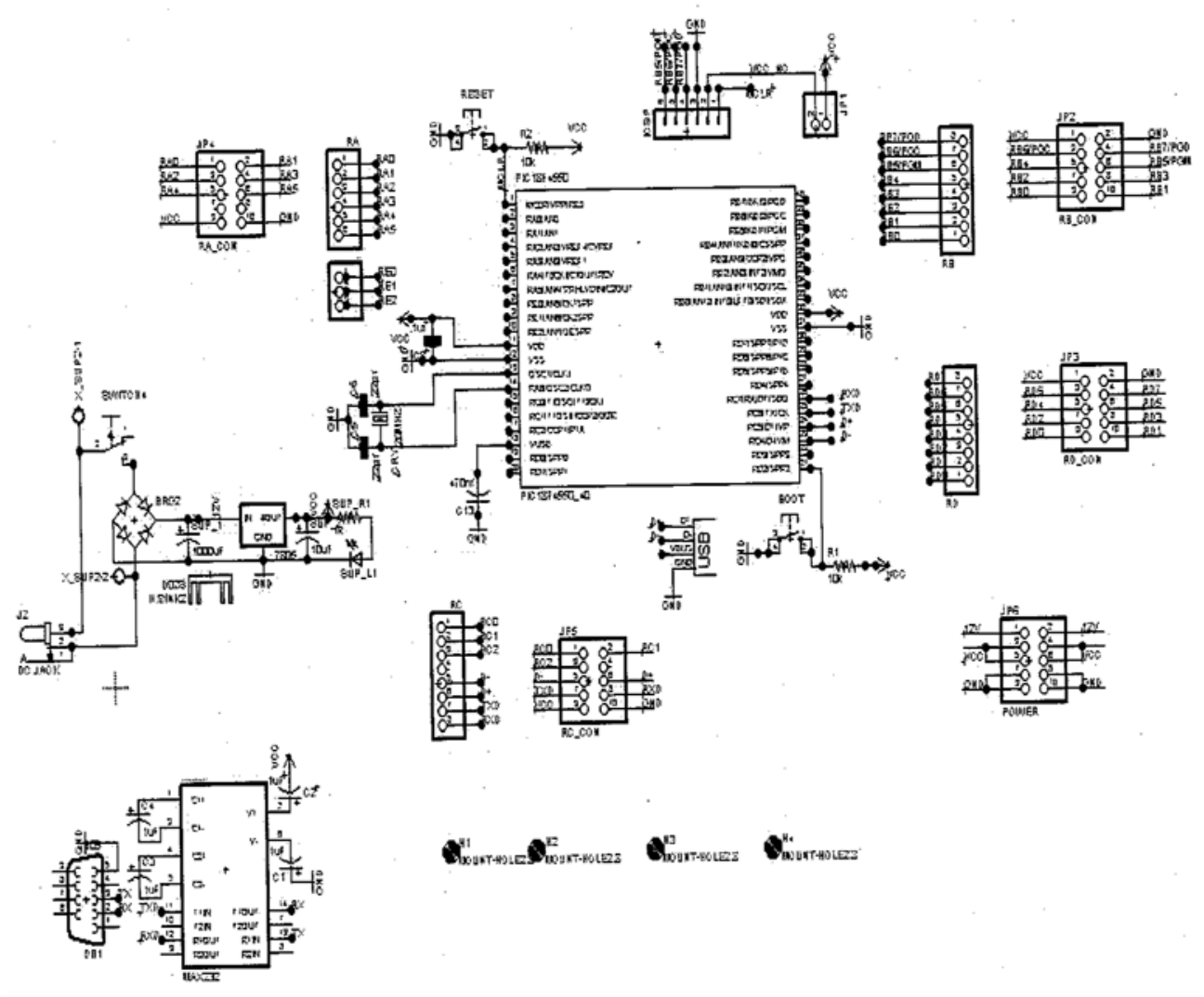

Fig. 6 Circuit Diagram of PIC

\subsection{Motor driver L293D}

DC motors are operated on $12 \mathrm{~V}$ supply. Microcontroller 8051 is not capable of providing such a high voltage. It require motor driver to overcome this problem. There are three drivers available-ULN2803, L298 and L293D. In this study L293D driver is used because it is cost effective as compare to other and also it is compact in size. L293D is a monolithic integrated high voltage, high current for channel driver which designed to accept standard DTL or TTL logic levels and drive loads. The L293D is assembled in a 16 lead plastic has four centre pins connected together and used for heat sinking [6].

\subsection{Voltage regulator 7805}

Voltage regulator produces fixed DC output voltage from variable DC (a small amount of AC in it). Normally fixed output is getting by connecting the voltage regulator at the output of the filtered DC as shown in Fig. 07. It can also used in circuit to get low DC voltage from high DC voltage. In this work 7805 voltage regulator is used to get supply of $5 \mathrm{~V}$ from $12 \mathrm{~V}$. $78 \mathrm{XX}$ is most commonly used series of voltage regulator, but in this research 7805 voltage regulator is used because it gives fixed $5 \mathrm{~V}$ DC voltage for input voltage range of $7.5 \mathrm{~V}$ to $20 \mathrm{~V}$.

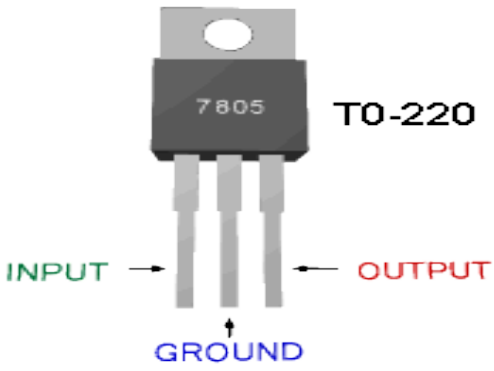

Fig. 7 Voltage Regulator 


\subsection{MAX 232}

The MAX232 family of line driver or receiver is intended for all EIA/TIA 232E and V.28/V.24 communication interface. These parts especially useful in battery power system, since its low power shutdown mode reduces power dissipation to less than 5V. MAX 232 needs external capacitors for the internal voltage pump.

\subsection{RF Transmitter/RF Receiver}

In the present vehicle, TX4915-F transmitter is used for modulation. The TX4915-F is an entire PhaseLocked Loop (PLL) for precise local Oscillator generation. It can use in OOK / HCS / PWM signal and modulate to transmit signal to receiver device by air .It had a high performance and easily to design your product. In present study, RF transmitter TX4915 is used for specific remote-control function. It supports $315 \mathrm{MHz} / 433.92 \mathrm{MHz} / 868.35 \mathrm{MHz} / 914.5 \mathrm{MHz}$ for ISM Band modules. In receiver section of this vehicle, the RX3400 is a Miniature receiver module that receives FSK Modulation Signal and demodulated to digital signal for the next decoder stage. Local Oscillator is made of PLL structure. Since RX3400 is designed specifically for remote-control and wireless security receiver operating at $315 \mathrm{MHz} / 433.92 \mathrm{MHz}$, in this research it is used in remote control section. The circuit diagram for RF transmitter/RF receiver used in this work is as shown in Fig. 08 .

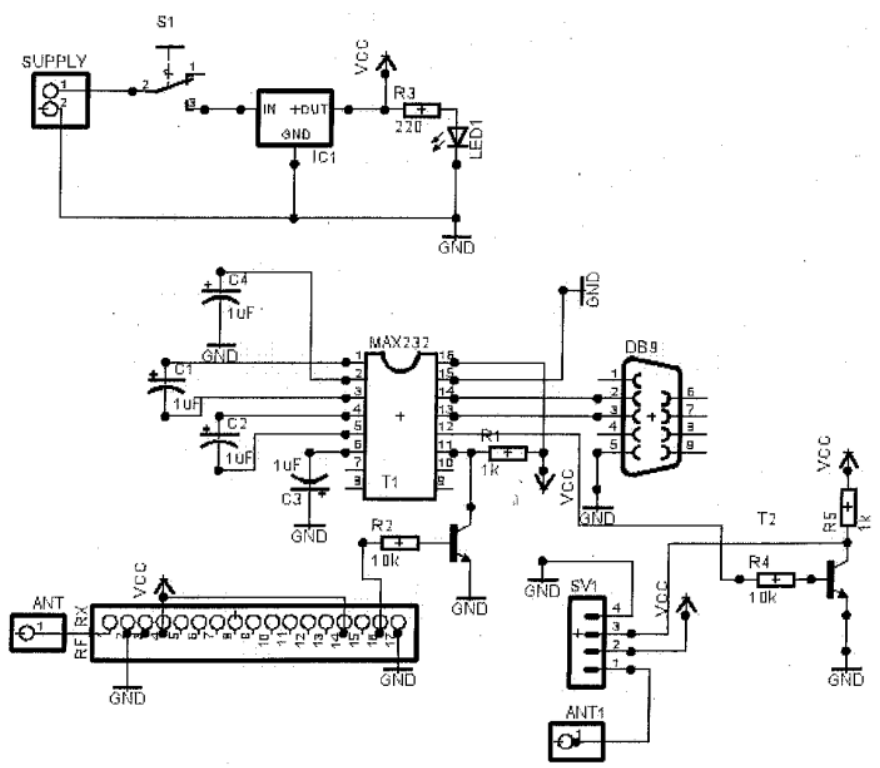

Fig. 8 Circuit diagram of RF Transmitter/RF Receiver

\section{TOUCH SCREEN INTERFACING WITH PIC}

In the present study touch screen is interfaced with Programmable Intercombination Circuit (PIC 18F4550). Figure 09 shows touch screen interfacing with PIC. It indicates touch screen having four channels interfaced with PIC PIN. Out of these four channels, channels 1 and 2 are digital pins whereas channel 3 and 4 are analog pins. Pins RB0, RB1, RB2, and RB3 are connected to channel 1, 2, 3 and 4 respectively. Pin RB2 and RB3 which are nothing but AN8 and AN9 (analog pin) are interfaced with channel 3, 4 to take analog input from touch screen $[7,8]$. 


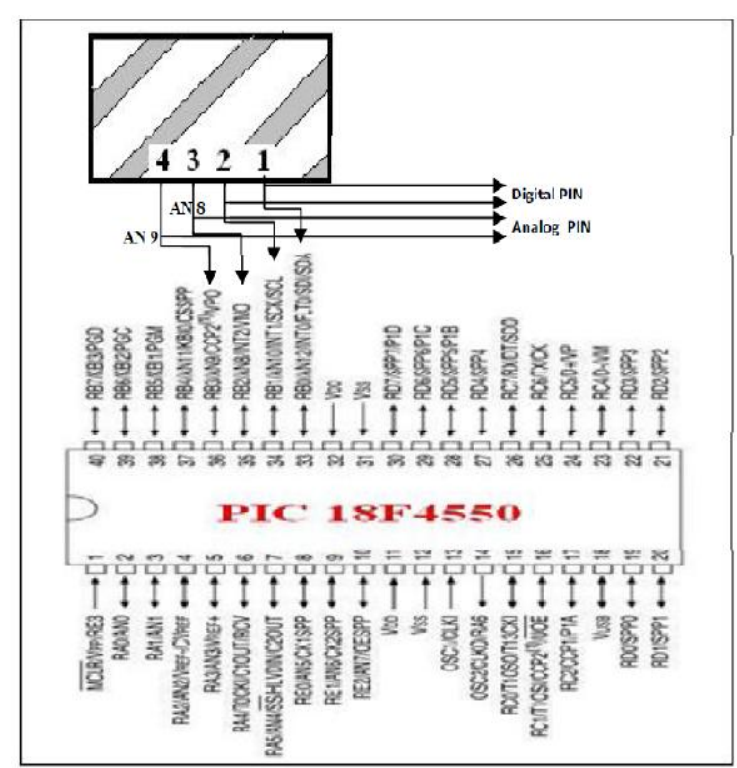

Fig. 9 Touch screen interfacing with PIC 18F4550

This interfacing configuration of port B is explained in following Table No. 03.This provides information regarding three major modes which are touch detection, $\mathrm{X}$ channel detection and $\mathrm{Y}$ channel detection. When PIN 3 and 4 are constant and PIN 2 is connected to digital input of PIC then it started sensing of touch detection. Similarly, PIN 3 is connected to AN8 and PIN 4 is connected to $+5 \mathrm{~V}$ then it detects X channel whereas PIN 1 is connected to $+5 \mathrm{~V}$ and PIN 4 is connected to AN9 then it detects Y channel.

Table No. 03 Port B Configuration

\begin{tabular}{lcccc}
\hline \multicolumn{1}{c}{ Function } & $\begin{array}{c}\text { RB0 PIN 1 } \\
\text { Touch Screen }\end{array}$ & $\begin{array}{c}\text { RB1 PIN 2 } \\
\text { Touch Screen }\end{array}$ & $\begin{array}{c}\text { RB2 PIN 3 } \\
\text { Touch Screen }\end{array}$ & $\begin{array}{c}\text { RB3 PIN 4 } \\
\text { Touch Screen }\end{array}$ \\
\hline Touch & $\mathbf{0}$ & Digital input & NC & NC \\
Xetection & NC Channel & $\mathbf{0}$ & AN 8 & $+\mathbf{5 ~ V}$ \\
Y_Channel & $\mathbf{+ 5}$ V & NC & $\mathbf{0}$ & AN 9 \\
\hline
\end{tabular}

The touch detection is schematically represented as given Figure 10.

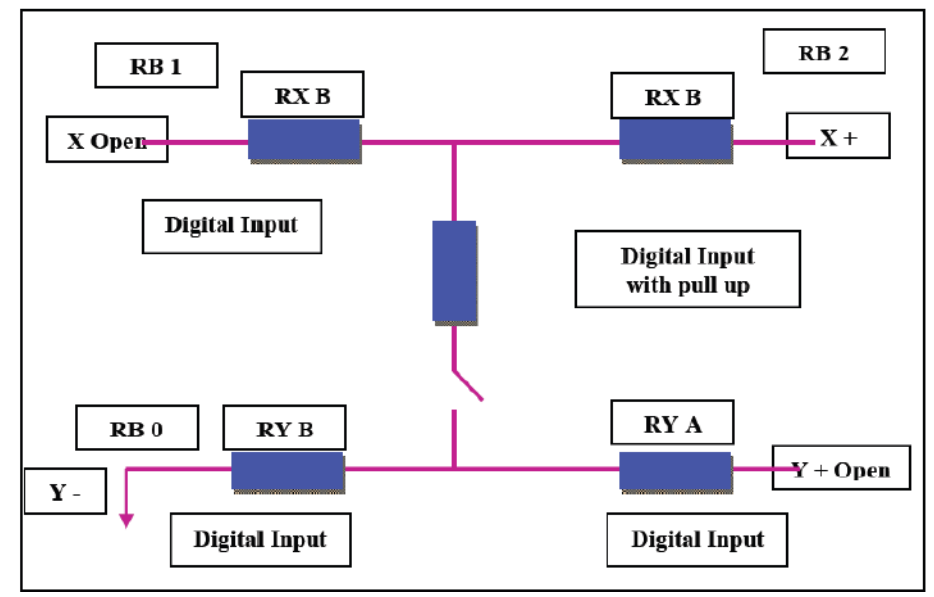

Fig. 10 Touch Detection

\section{TOUCH SCREEN PROGRAMMING}

In this study, Keil software is used for touch screen programming. The Keil software is C compiler software. It converts $C$ language into the machine language. The program which executes motion of touch pad controlled vehicle is as given below. Program consist five different positions of vehicle; those are left, right, 
forward (top), backwards (bottom) and center. In this program entire touch screen is divided into X mode and $\mathrm{Y}$ mode. These two modes decide different positions of vehicle and on the basis of these positions program is made through which vehicle gets appropriate motion [9].

\# include<p18f4559.h>

\#include"delay.h"

\#include"ADC.h"

\#define DEBUG

\#define to $\operatorname{Ascii}(x)(x+0 x 30)$

//Touch screen

\#define X MAX

\#define Y_MAX

\#define X_MIN

\#define Y_MAX

\#define X_MID

\#define Y_MID

\#define BOX_LENGTH

\#define LEN

\#define SEPARATOR

//CENTER

\#define CENTER_BOTTOM_LEFT_X \#define CENTER_BOTTOM_LEFT_Y

\#define CENTER_BOTTOM_RIGHT_X

\#define CENTER_BOTTOM_RIGHT_X

\#define CENTER_TOP_LEFT_X

\#define CENTER_TOP_LEFT_Y

\#define CENTER_TOP_RIGHT_X

\#define CENTER_YOP_RIGHT_Y

\section{//RIGHT}

\#define RIGHT_BOTTOM_LEFT_X

(LEN+SEPARATOR+BOX_LENGTH)

\#define RIGHT_BOTTOM_LEFT_Y

\#define RIGHT_BOTTOM_RIGHT_X

\#define RIGHT_BOTTOM_LEFT_Y

\#define RIGHT_TOP_LEFT_X

\#define RIGHT_TOP_LEFT_Y

\#\#define RIGHT_TOP_RIGHT_X

define RIGHT_TOP_RIGHT_Y

// LEFT

\#define LEFT_BOTTOM_LEFT_X

\#define LEFT_BOTTOM_LEFT_Y

\#define LEFT_BOTTOM_RIGHT_X

(LEN+SEPARATOR+BOX_LENGTH)

\#define LEFT_BOTTOM_RIGHT_Y

\#define LEFT_TOP_LEFT $X$

\#define LEFT_TOP_LEFT_Y

\#define LEFT_TOP_RIGHT_X

(SEPARATOR+BOX_LENGTH)

\#define LEFT_TOP_RIGHT_Y

I/TOP

\#define TOP_BOTTOM_LEFT_X

\#define TOP_BOTTOM_LEFT_Y

\#define TOP_BOTTOM_RIGHT_X

\#define TOP_BOTTOM_RIGHT_Y

\#define TOP_TOP_LEFT_X

\#define TOP_TOP_LEFT_Y

\#define TOP TOP RIGHT $X$

\#define TOP_TOP_RIGHT_Y

//BOTTOM

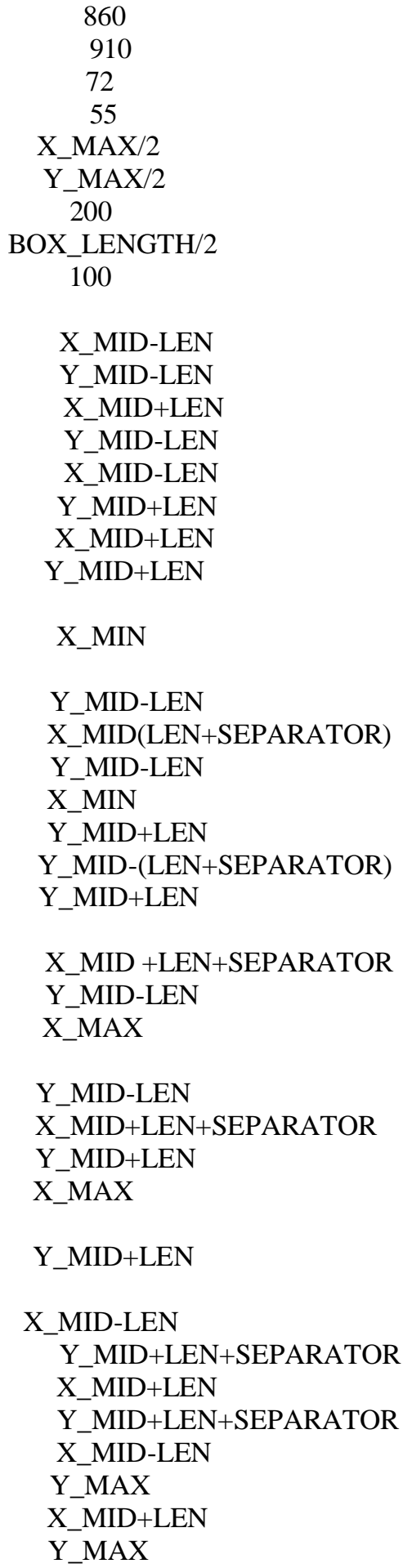




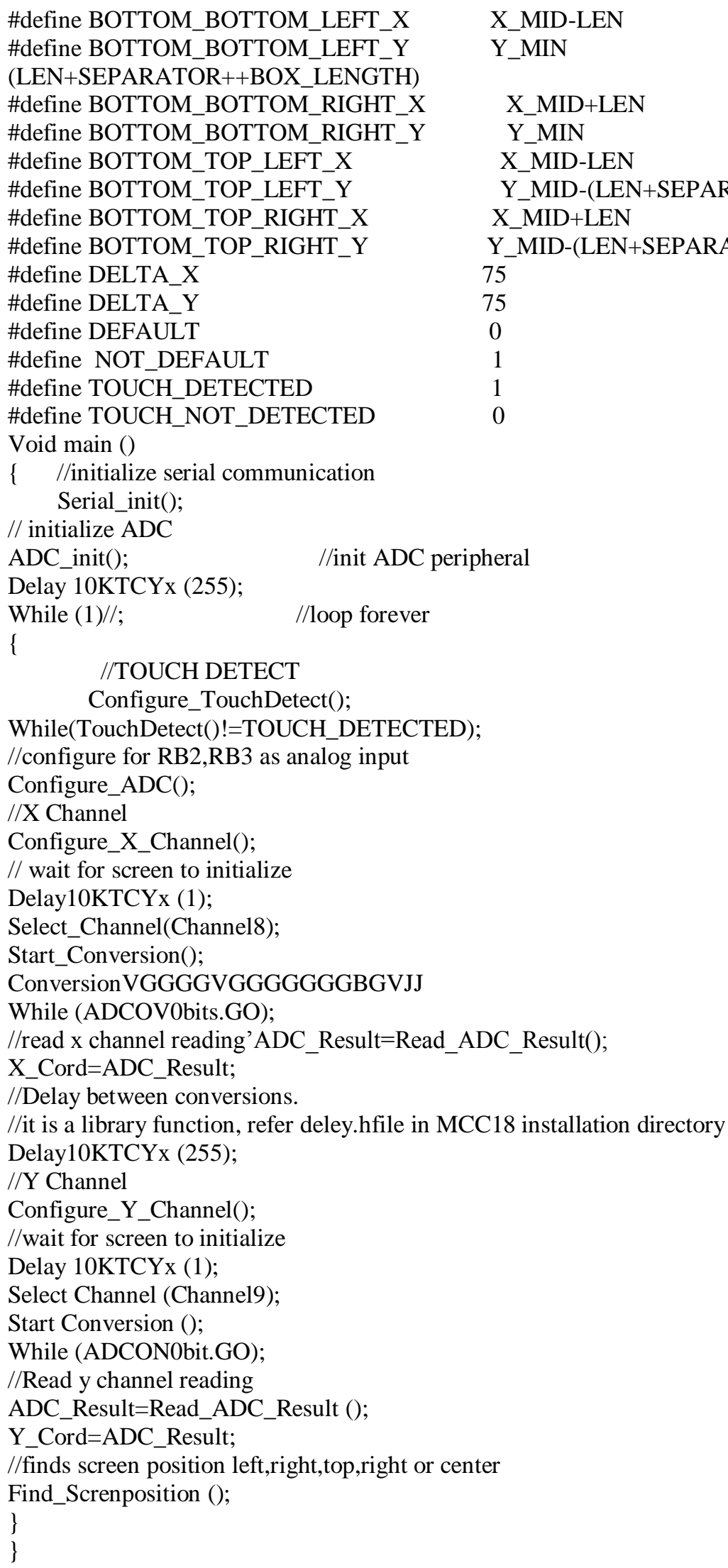

\section{CONCLUSION}

In this current study, performance of vehicle is evaluated by taking number of test drives. It is found that vehicle reaches to appropriate position as per instructed by touchpad. Followings are major conclusions. 
- Moving capacity of this vehicle is totally dependent of capacity of RF transmitter and Receiver. In the present study it was found to be 12 - 16 meters. High quality, high capacity RF transmitter and Receiver provide more moving area.

- Programmable Intercombination Controller PIC 18F4550 is found to be suitable for this application because it inbuilt programmable flash memory.

- It is observed that L293 D motor driver is effectual as it works even for least power supply of $+5 \mathrm{~V}$ to drive vehicle.

- This present model can be used for wild life discover just by attaching micro-camera on it for example to study living style of snake, tiger etc. This can be also used in critical situations like identifying exact location of hidden terrorist, enemy.

- It can be used where reaching of human being is practically not possible and fulfill all required activity just by adding appropriate high quality sensors on it.

\section{ACKNOWLEDGMENTS}

The authors gratefully acknowledge the great support from SSPM college of Engineering, Kankavli, Sindhudurg. Also, the authors would like to thank to the Mumbai University for support.

\section{REFERENCES}

[1] Brian W. Grasby, "A smarter computer controlled model car”, Monash University School of Computer Science and Software Engineering, 2002.

[2] Landauer, T. K., Nachbar, D. W. "Selection from Alphabetic end Numeric Menu Treea Using A Touch Screen: Breadth, Depth, and Width", Bell Communications Research Morristown, N.J. 1985.

[3] Vilimek, R., Zimmer, A. "Development and Evaluation of a Multimodal Touchpad for Advanced In-Vehicle Systems", Engin. Psychol. and Cog. Ergonomics, HCII 2007, Springer- Verlag Berlin Heidelberg, 2007.

[4] H. W. Huang, “ Design and Implementation of Electronic Control Trainer with PIC Microcontroller ”, Intelligent Control and Automation, Vol. 3 No. 3, 222-228,2012.

H. Rongen, "Introduction to PIC Microcontroller", Forschungszentrum Jülich Zentrallabor für Elektronik, Jülich, 2009.

[5] Johansson, H. \& Walter, K. "InVehicle Screen-Density, Driver distraction and User Preferences for Low vs. High Screen Density in intergrated Display”, Linkoping's Universitet, Sweden, 2005.

[6] Rydström, A., Broström, R. \& Bengtsson, P. "Safety aspects of using multifunctional control interfaces while driving - a simulator", 2008.

[7] H. W. Huang, "PIC Microcontroller: An Introduction to Software and Hardware Interfacing,", 2005.

[8] Keil, M., Mixon, R., Saarinen, T., and Tuunainen, V., "Understanding Runaway IT Projects: Results from an International Research Program Based on Escalation Theory," Journal of Management Information Systems, Vol. 11, No. 3, 67-97, 1995. 